\title{
A Centripetal Acceleration Statistic for Tracking Maneuvering Targets with Radar
}

\author{
David Bizup \\ University of Virginia \\ Department of Systems and Information Engineering \\ P.O. Box 400747 \\ 151 Engineer's Way \\ Charlottesville, VA 22904 \\ dfb2ph@virginia.edu
}

\begin{abstract}
Maneuver tracking is complicated because radar cannot directly measure target acceleration. Radar measures a target's range and bearing, and its radial velocity with respect to radar position. Tracking algorithms use radial velocity to associate radar reports to tracks, but not to update track state estimates. In this paper, a new statistic of centripetal acceleration based on the radial velocity measurement is developed. A tracker using this statistic is developed and tested via simulation against three other tracking algorithms. Simulation results show that the new tracker outperforms trackers with switched noise levels and a two mode interacting multiple model.
\end{abstract}

\section{Introduction}

The radar target tracking process involves measurement, association, and filtering. Radar measures target range and bearing. Some radars, using the Doppler shift of the returned signal, also measure target velocity or range rate along the line extending from the radar to the target. The radial velocity measurement $k_{k}$ is frequently used to associate contacts to existing tracks [Kameda et al., 2002]. It is not used to update track state estimates. Trackers that ignore rk during the filtering phase are not using all the available information about the track state. The reason is simple: radial velocity is non-monotonic with respect to the state space, and so highly nonlinear that the usual techniques for handling nonlinear measurements, like the Extended Kalman Filter (EKF), fail to provide satisfactory results.

Multisensor, multitarget data fusion systems (MMDFs) use filtered and unfiltered sensor data from radar and other sensors to produce situational information. Some sensors provide unfiltered reports that are associated with other reports and tracks. Some sensors generate tracks using only their own data. MMDFs associate these tracks to other tracks in the system. Good tracking algorithms, especially those that track maneuvers well, increase the chance of correct association and overall situation awareness.

Maneuver tracking is an important and particularly difficult task. The maneuver must be detected, then the track filter must respond appropriately. Interacting Multiple Model (IMM) filters, input estimation, and switched noise level filters [Schutz, et al., 1999] are used to track maneuvering targets. IMMs have two or more filters, each modeling a different maneuver. The 
filters run independently, and their outputs are combined as a weighted sum, where the weights are proportional to the relative likelihoods that each mode best represents the true target behavior. For example, the two mode IMM in [Bar-Shalom and Li, 1995] has a uniform motion, low noise model and a turning, high noise model. Input estimation techniques [Lee and Tahk, 1999] monitor the position measurement error sequence and estimate the accelerations causing a maneuver. The estimates are then used to project the target forward in time. Switched noise level trackers [Schutz, et al., 1999] monitor the position measurement error sequence and, based on statistical trend and threshold tests, choose between high and low noise levels. Thus, the position measurements are used twice: once to detect or estimate a maneuver, then again to update the state estimate.

None of the usual algorithms for tracking in two or three dimensions use to filter the state estimate. A range only tracker [Ramachandra, 1993], with track state vector $\left[\begin{array}{ll}x & x\end{array}\right]$, can use $k_{k}$ because it is a direct, linear measurement of the second state variable. It is significant that none of the higher dimensional trackers in [McIntyre and Hintz, 1998] or [Ramachandra, 2000] use $*$. [Kosuge, et al., 1998] propose a track filter that rotates the coordinate frame so that one of the axes is in the direction of the radial to the target. Then, $k$ is a linear measurement of one state variable and is used in a Kalman Filter (KF) tracker. After updating, the state estimate is rotated back to the original frame.

This paper develops a new statistic of centripetal acceleration based on the radial velocity measurement $k$. It detects maneuvers and estimates their magnitude. A tracker using the centripetal acceleration statistic is developed and tested via simulation against a uniform motion KF tracker, a switched noise tracker, and a two mode IMM. The new tracker outperforms the others on all significant measures, including position speed errors during a maneuver and convergence back to the true value when the maneuver ends. It is less computationally intensive than the IMM.

\section{Problem Formulation}

The problem is formulated as a linear system in a rectangular, $x-y$, coordinate frame as shown in figure 1. The positive $x$ axis is east, and the positive $y$ axis is north. A rotating radar measuring range $r_{k} r$, bearing $\theta_{k}$ and radial velocity r $_{k}$ is fixed at the origin. Range and bearing are converted to pseudo-measurement $\mathbf{z}_{k}$ by

$$
\mathbf{z}_{k}=\left[\begin{array}{c}
\tilde{x}_{k} \\
\tilde{y}_{k}
\end{array}\right]=\left[\begin{array}{c}
r_{k} \cdot \sin \theta_{k} \\
r_{k} \cdot \cos \theta_{k}
\end{array}\right]
$$

If the measurement noise is Gaussian, $\mathbf{z}_{k}$ is approximately Gaussian and linear. A vector of positions and velocities $\mathbf{x}_{k}=\left[\begin{array}{llll}x_{k} & y_{k} & y_{k}\end{array}\right]^{T}$ describes the track state. This coordinate system is natural and effective for the converted position measurements and target dynamics. Unfortunately, is non-monotonic and highly nonlinear in this frame. 
Pseudo-measurements $\mathbf{z}_{k}$ update track state in a uniform motion KF tracker with state and measurement equations

$$
\begin{gathered}
\mathbf{x}_{k+1}=F \mathbf{x}_{k}+G \mathbf{w}_{k} \\
\mathbf{z}_{k}=H \mathbf{x}_{k}+\mathbf{v}_{k} \\
F=\left[\begin{array}{cccc}
1 & 0 & T & 0 \\
0 & 1 & 0 & T \\
0 & 0 & 1 & 0 \\
0 & 0 & 0 & 1
\end{array}\right], G=\left[\begin{array}{cc}
T^{2} / 2 & 0 \\
0 & T^{2} / 2 \\
1 & 0 \\
0 & 1
\end{array}\right], H=\left[\begin{array}{cccc}
1 & 0 & 0 & 0 \\
0 & 1 & 0 & 0
\end{array}\right] . \mathbf{w}_{k}=\left[\begin{array}{l}
w_{x} \\
w_{y}
\end{array}\right] \text { is a vector of } \\
\text { zero-mean, Gaussian accelerations with covariance } Q_{k} \text {. The measurement noise } \mathbf{v}_{k}=\left[\begin{array}{l}
v_{x} \\
v_{y}
\end{array}\right] \text { is }
\end{gathered}
$$
zero-mean Gaussian with covariance $R_{k}$ that depends on the measurements and their noises. $T$ is the time between measurements. When (1) accurately represents the true system dynamics then the tracker produces good estimates. Uniform target motion along a straight line is modeled by making $Q_{k}$ small. When $Q_{k}$ is small the gain is low and the measurement does not change the state estimate very much. Maneuvers are modeled by making $Q_{k}$ large. When $Q_{k}$ is large the gain is high and the measurement is heavily weighted. The most common difficulty is the specification of the covariance matrices $Q_{k}$.

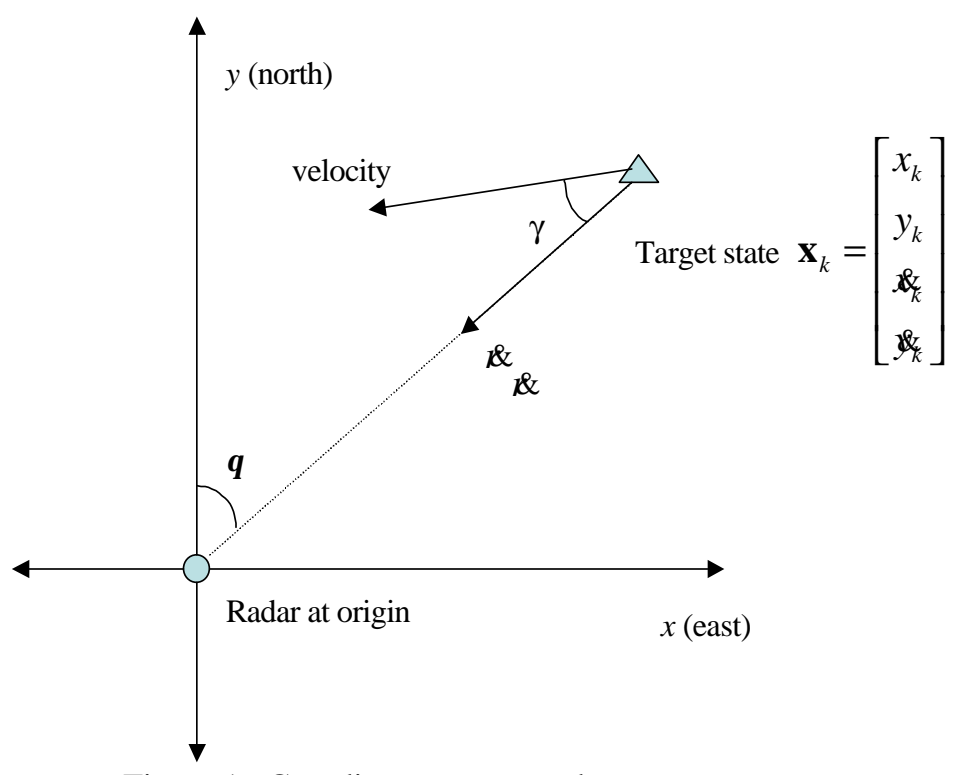

Figure 1. Coordinate system and target state vector 


\subsection{The Trouble With Radial Velocity}

Notice that $k$ plays no part in the preceding formulation. There are two problems with in the $x-y$ coordinate system. First, it is highly nonlinear. Second, it has no unique inverse. In figure $1, \gamma$ is the angular difference between target heading and bearing. Radial velocity is the negative product of speed and $\cos (\gamma)$. Rewriting the cosine in terms of the state variables yields the following expression for radial velocity.

$$
\text { radial_velocity }=-\sqrt{x^{2}+y^{2}} \cdot \cos (\gamma)=\frac{x \cdot x+y \cdot y}{\sqrt{x^{2}+y^{2}}}
$$

Clearly, radial velocity is both nonlinear and non-monotonic with respect to the state variables. In fact, it is so highly nonlinear that the usual technique of approximating it by a linear function in an EKF does not work. The approximate linear equation is a function of the first order partial derivatives. The denominator of (2) is the range. So, when the target is moving nearly tangentially to the radar, the range rate of change is nearly zero and the partial derivatives can be very large. An EKF tracker using was reported in [Schutz, et al., 1997] to diverge for most target trajectories.

It is also easy to see that there is no unique, closed form inverse between radial velocity and the elements of $\mathbf{x}$. The state vector has four elements, just one. For every $k_{k}^{k},(2)$ has an infinite number of solutions. Fix any one or two elements of $\mathbf{x}$, and (2) still has an infinite number of solutions. Therefore, even a perfect, noiseless radial velocity measurement is not much use in updating the state estimate.

\section{Developing A Centripetal Acceleration Statistic Using Radial Velocity}

Maneuvers are modeled as turns with a constant but unknown rate that last for the entire interval between measurements. By conditioning on the previous state estimate and most recent the set of allowable turns is restricted. Each turn corresponds to a centripetal acceleration with constant magnitude. The minimum acceleration is a statistic that turns out to be a reliable indicator that the target is maneuvering.

\subsection{Maneuver Model}

Conditional on the previous state estimate, assume that the target either does not maneuver or travels at constant speed along a circular arc for the entire interval between measurements, as shown in figure 2. Its heading at every instant is tangential to the arc. The time between measurements is known, so the distance traveled is known. For an arc of given radius, the target's position, speed, and heading are known at all times in the interval. In particular, they are known at the time of the most recent measurement. Knowing these quantities and the radar location is sufficient to determine the radial velocity. 


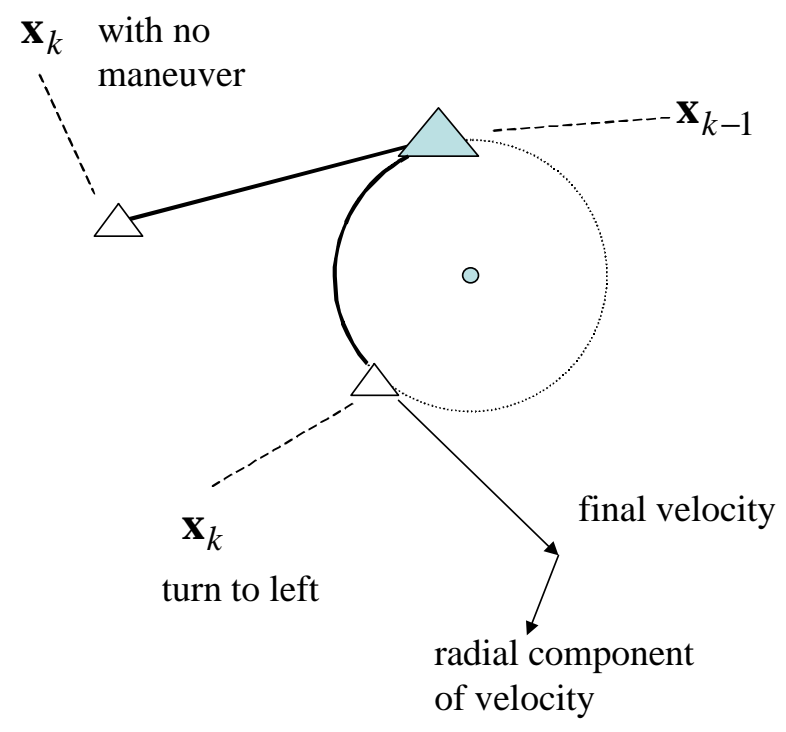

Figure 2. Maneuver model for some left turn

Not every turn is allowed. Only those such that the target's final radial velocity equals or are allowed. Recall from figure 1 that radial velocity equals the product of speed and $\cos (\gamma)$, the angular difference between heading and bearing. Cosine is a symmetric function so there are two possible final headings: one to the left of the bearing line and one to the right. These are denoted $h_{0}$ and $h_{1}$ in figure 3 . Headings $h_{0}$ and $h_{1}$ can be realized by turning to the right or left, so there are four possible turns.

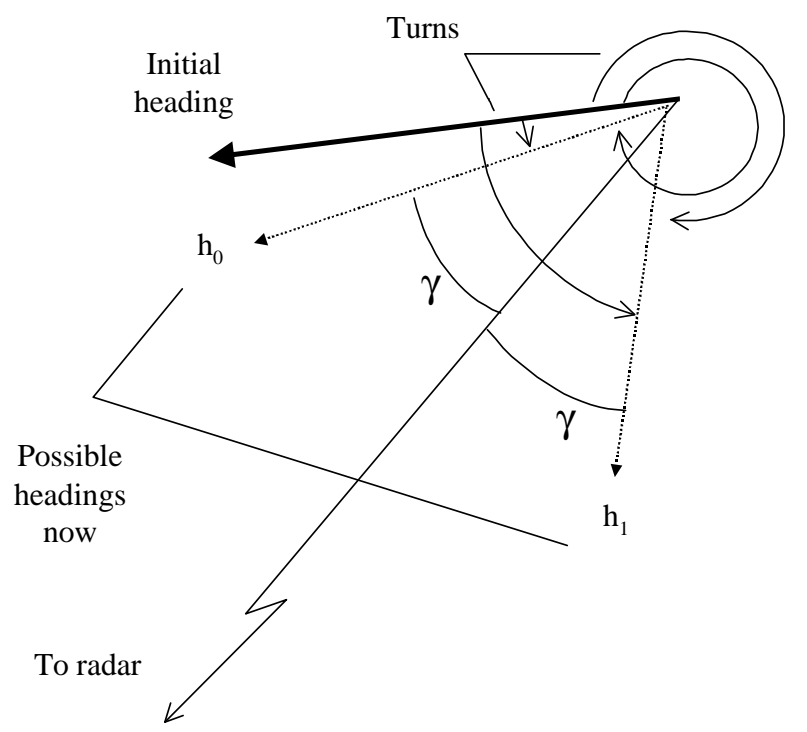

Figure 3. Four possible turns 


\subsection{Calculating the Statistic}

Let $\theta_{k}$ denote the bearing to the target, and $\eta_{k}$ the target heading. If the range to the target is large and the time between measurements is small, then the bearing will not change much and $\theta_{k} \approx \theta_{k-1}$. These quantities are calculated in (3).

$$
\begin{gathered}
\theta_{k-1}=\tan ^{-1}\left(x_{k-1} / y_{k-1}\right) \\
\eta_{k-1}=\tan ^{-1}\left(x_{k-1} / x_{k-1}\right)
\end{gathered}
$$

Headings $\mathrm{h} 0$ and $\mathrm{h} 1$ are calculated in (4).

$$
\begin{gathered}
\gamma=\cos ^{-1}\left(-k_{k} / \sqrt{x_{k-1}^{2}+x_{k-1}^{2}}\right) \\
h_{0}=-\theta_{k}+\gamma, \quad h_{1}=-\theta_{k}-\gamma
\end{gathered}
$$

Let ô denote the magnitudes of the four possible heading changes.

$$
\hat{\mathbf{o}}=\left\{ \pm\left(\eta_{k-1}-h_{0}\right), \pm\left(\eta_{k-1}-h_{1}\right)\right\} \bmod 2 \pi
$$

The length of the arc defining the path equals distance traveled, $d$. Angular arc width equals heading change. The radii of the possible paths equal the ratio of their angular widths to their lengths, $\hat{\mathbf{o}} / d$. Centripetal accelerations causing circular motion equal the ratio of the square of speed to radius. The accelerations corresponding to each turn can be calculated. The minimum acceleration is a statistic of centripetal accelerations that turns out to be a reliable indicator of a target maneuver.

$$
\begin{aligned}
\mathbf{c}=\text { speed }^{2} / \text { radius } & =\left(k_{k-1}^{2}+k_{k-1}^{2}\right) \cdot d / \hat{\mathbf{o}} \\
c_{\min } & =\min (\mathbf{c})
\end{aligned}
$$

\subsection{Evaluating the Statistic via Simulation}

$c_{\text {min }}$ was evaluated via simulation using a uniform motion $\mathrm{KF}$ tracker having state noise covariance matrix $q^{2} \mathbf{I}$, where $q$ equals $0.03 \mathrm{~g}$. The radar rotated at $6 \mathrm{rpm}$, was fixed at the origin and had range, bearing and $z$ measurement standard deviations of 100', 0.1 radian, and 2 knots, respectively. The intent was to simulate a rotating, air traffic control radar. The target moved at constant speed and made turns of specified centripetal acceleration. Target speed was varied from 300 to $600 \mathrm{knots}$, and the turns from $1 \mathrm{~g}$ to $6 \mathrm{~g}$ 's, where $1 \mathrm{~g}$ equals $9.8 \mathrm{~m} / \mathrm{sec}^{2}$. The results were used to map $c_{\min }$ into likelihood functions of a maneuver, conditional on the acceleration. A more complete analysis is required to form the likelihoods over the whole range of target speeds and accelerations of interest. The simulation results showed that centripetal acceleration statistic $c_{\min }$ is a reliable indicator of a maneuver. 


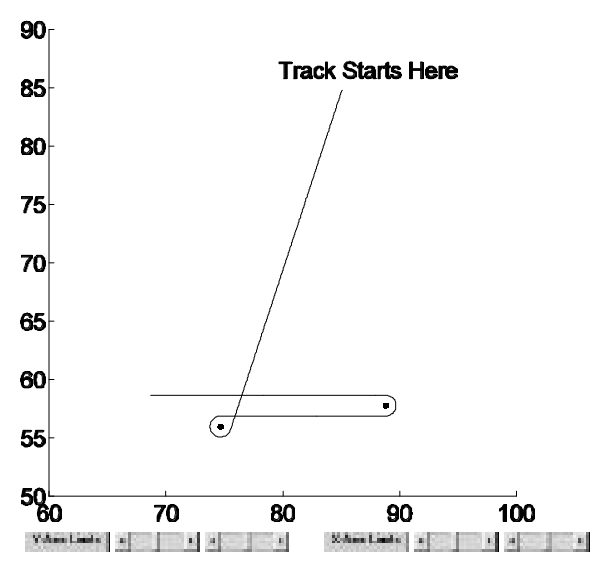

Figure 4. Sample Target Trajectory

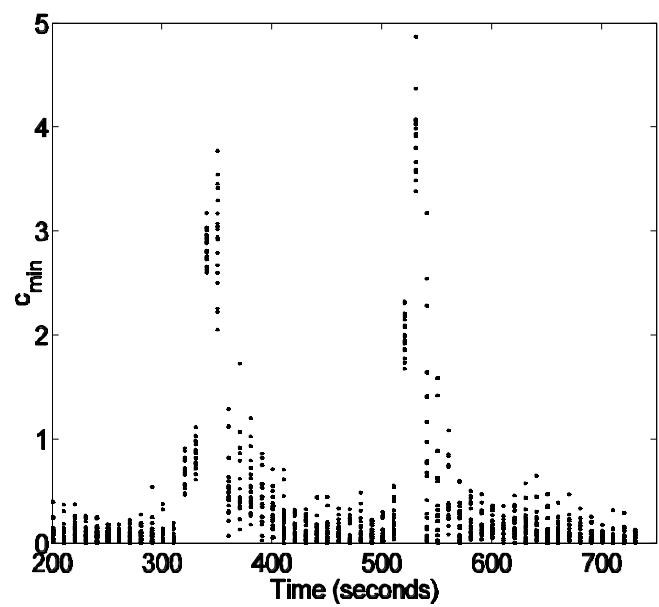

Figure 5. $c_{\min } \mathrm{v}$ Time, for 20 runs

Figures 4 shows the target trajectory from one scenario used to evaluate $c_{\min }$. A 350 knot target started about 120 miles away and made two $2 \mathrm{~g}$ turns. The first turn lasts about 40 seconds, the second about 30. This scenario was run 20 times. Figure 5 shows the value of $c_{\min }$ at each update for each run. Clearly, $c_{\min }$ is a good indicator of a maneuver: it is high during and just after a maneuver, and low otherwise. Were a maneuver declared whenever $c_{\text {min }}$ exceeded $0.5 \mathrm{~g}$, the first maneuver would always be detected immediately and the second maneuver would always be detected within two scans. The false alarm rate is low. In this scenario, there are 9 false alarms in 20 runs, with 73 track updates in each run. The false alarm rate is only 0.006 per track state update.

\section{Tracking with $\mathbf{c}_{\min }$}

Simulation results show that $c_{\text {min }}$ reliably indicates a maneuver. A tracker that exploits this property is developed and tested. The tracker is a uniform motion KF with state and measurement equations (1). The state noise variance $Q$ equals $(0.03 \mathrm{~g})^{2} \mathbf{I}$ whenever $c_{\min }$ is less than $1 \mathrm{~g}$, and equals $\left(c_{\min }\right)^{2} \mathbf{I}$ otherwise.

\section{$4.1 \quad$ Simulation Results}

A simulation is used to compare the performance of the new tracker described in section 3.3 to three other tracking algorithms. The first is a uniform motion KF tracker with constant state noise variance $Q$ equal to $(0.03 \mathrm{~g})^{2} \mathbf{I}$. This is the baseline tracker. The second [Schutz, et al., 1999] is a Combined KF (CKF) that switches between two noise levels based on a threshold test of the fading memory average (FMA) of the radar position errors. The FMA decay rate is .1. If the FMA is less than 5 miles then $Q$ is set to a low $(0.03 \mathrm{~g})^{2} \mathbf{I}$, otherwise $Q$ is set to $(2 \mathrm{~g})^{2} \mathbf{I}$. The final tracker is a two mode Interacting Multiple Model (IMM). One mode has low state noise of $(0.03 \mathrm{~g})^{2} \mathbf{I}$ representing uniform motion. The other has a high noise of $(2 \mathrm{~g})^{2} \mathbf{I}$ representing a maneuver. IMM output equals a weighted sum of the outputs of the individual models. The weights are proportional to the product of the likelihoods and the a priori probabilities. In this test, the a priori probability of a maneuver is always 0.1 . 
Table 1. Four tracking algorithms tested via simulation

\begin{tabular}{|c|c|c|}
\hline Tracker & Description & Based on \\
\hline $\begin{array}{l}\text { Kalman Filter }(\mathrm{KF}) \\
\text { using } c_{\min }\end{array}$ & $\begin{array}{l}\text { Four state KF radar tracker as in equation }(1) \text {, } \\
\text { using } c_{\min } \text { to set the gain } Q \text {. } \\
\text { If } c_{\min }<1 \mathrm{~g} \text {, then } Q=(0.03 \mathrm{~g})^{2} \mathbf{I} \text {, } \\
\text { Otherwise, } Q=\left(c_{\min }\right)^{2} \mathbf{I} \text {. }\end{array}$ & This paper. \\
\hline Uniform Motion KF & $\begin{array}{l}\text { Four state KF radar tracker as in equation (1) } \\
Q=(0.03 \mathrm{~g})^{2} \mathbf{I} \text {. }\end{array}$ & [Bar-Shalom and Li, 1995] \\
\hline $\begin{array}{l}\text { Combined KF } \\
(\mathrm{CKF})\end{array}$ & $\begin{array}{l}\text { KF radar tracker as in equation }(1) \text {, using FMA to } \\
\text { switch noise levels. FMA decay rate }=0.1 \text {. } \\
\text { If FMA }<5 \text { miles, then } Q=(0.03 \mathrm{~g})^{2} \mathbf{I} \text {, } \\
\text { Otherwise, } Q=(2 \mathrm{~g})^{2} \mathbf{I} \text {. }\end{array}$ & [Schutz, et al., 1997] \\
\hline $\begin{array}{l}\text { Interacting Multiple } \\
\text { Model (IMM) }\end{array}$ & $\begin{array}{l}\text { Two mode IMM. One low noise and one high } \\
\text { noise mode, each as in equation (1). } \\
Q_{\text {low }}=(0.03 \mathrm{~g})^{2} \mathbf{I}, Q_{\text {high }}=(2 \mathrm{~g})^{2} \mathbf{I}\end{array}$ & [Bar-Shalom and Li, 1995] \\
\hline
\end{tabular}

All tracks start the same way. They initialize using a two hit rule. Then, for the next 12 detections, they are updated using a low noise KF. This gives them a chance to stabilize and allows a fair comparison apart from the effects of different startup policies. After that, the trackers work as described above. The radar is the same as in section 3.2. The target moves at a constant speed of 350 knots and makes $2 \mathrm{~g}$ turns. The whole scenario lasts for about 930 seconds, or 93 scans.

The simulation was run 100 times for the target trajectory shown in figure 6. Detection and association rates are perfect. The track is updated once per scan, or approximately once every ten seconds. The target's speed is constant at 350 knots, and it makes two, $2 \mathrm{~g}$ turns. The first turn occurs at about 290 seconds into the scenario, the second at about 610 . Figures 7 and 8 show the position and speed error means and $95 \%$ confidence bounds for the four trackers.

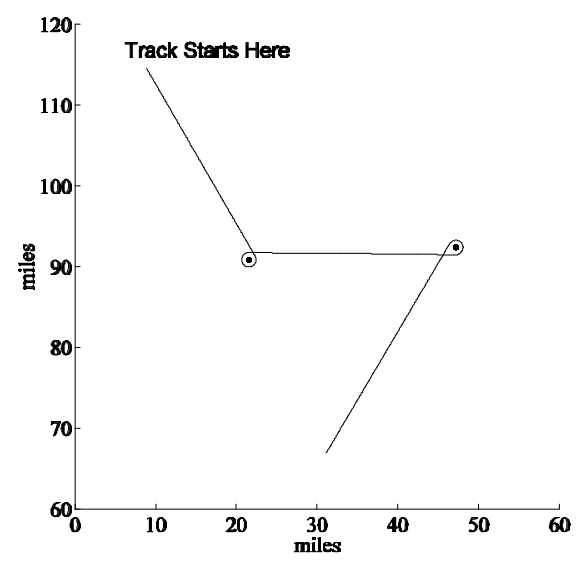

Figure 6. Target Trajectory 
In figure 7 , the mean position error of the tracker using $c_{\min }$ is about the same as the baseline tracker during the straight segments and is significantly better during the maneuver. This is the expected behavior. During straight line segments, $c_{\min }$ is low and the two trackers are identical. During maneuvers, $c_{\min }$ is large and the state noise is adjusted so that it is roughly proportional to the maneuver magnitude. Not only is position error lower during the maneuver, but it converges back to steady state faster when the maneuver ends. The switching CKF tracker exhibits the worst performance. The FMA test is slow to detect maneuvers, and causes more false alarms during the straight segments that $c_{\min }$. The CKF has only two noise levels, so when it declares a maneuver it cannot make the state noise variance proportional to the maneuver. Thus, false alarms degrade the track quality more than false alarms using that $c_{\text {min }}$. The IMM's mean performance is better than the CKF, but not quite as good as the new tracker. Interestingly, its error variance is about 50\% larger. This is because, like the CKF tracker, its models have only two noise levels. The model outputs are combined as a weighted sum, but if the wrong model is heavily weighted, then the track quality is significantly degraded.
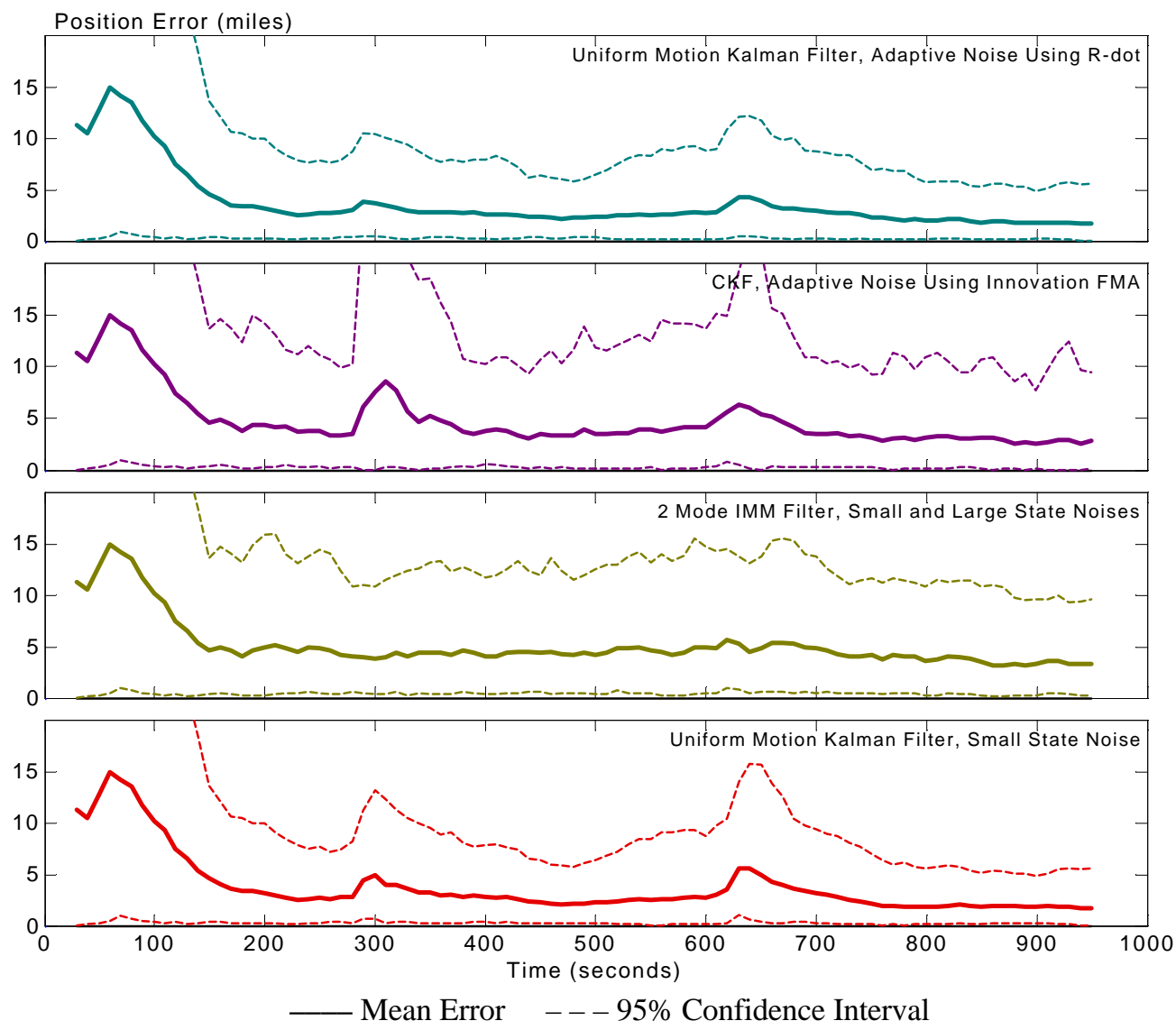

Figure 7. Position error versus time 
Similar results are seen for speed error in figure 8. The new tracker using $c_{\min }$ performs about the same as the baseline tracker during straight segments and better during maneuvers. It converges back to the true value in about one half the time after the first maneuver, but takes slightly longer to converge after the second maneuver. Its speed error mean and variance are considerably lower than the CKF or IMM. The CKF and IMM are clearly overcompensating for the maneuver. Once again, the CKF with switching noise variance is the worst performer. The IMM exhibits consistent mean error performance, but its variance is about $100 \%$ larger than the new tracker.

\section{Conclusion}

A new statistic of acceleration, $c_{\min }$, based on the radial velocity measurement $z^{2}$ is proposed in this paper. Most trackers ignore \& during track filtering because it is non-monotonic, highly nonlinear, and non-invertible in rectangular coordinate frames. Simulation results show that $c_{\min }$ is a reliable indicator of a target maneuver. A uniform motion KF tracker that uses $c_{\min }$ to set its state noise variance outperforms switched noise level and two mode IMM trackers in terms of position and speed error mean and variance, and convergence back to the true value when the maneuver ends. This tracker offers improved performance with a small change to the tracking algorithm and no change to the sensor. 


\section{6. $\quad$ References}

[Bar-Shalom and Li, 1995] Yaakov Bar-Shalom and Xiao-Rong Li, Multitarget-Multisensor Tracking, Principles and Techniques, YBS Publishing, 1995

[Kameda et al., 2002] Hiroshi Kameda, Shingo Tsujimichi, Yoshio Kosuge. Target Tracking Using Range Rate Measurements Under Dense Environments. Electronics and Communications in Japan, Part 1, Communications, volume 85, issue 3, 19-29, 20002.

[Kosuge, et al., 1998] Y. Kosuge, H. Kameda and S. Mano., "A decoupled filter for radar tracking with range rate measurements," Transactions of the Society of Instrument and Control Engineers, vol.34, no.5, pp.368-75, May 1998

[Lee and Tahk, 1999] Hungu Lee and Min-Jea Tahk, Generalized Input Estimation Techniques Technique for Tracking Maneuvering Targets, IEEE Transactions on Aerospace and Electronic Systems, Vol. 35, No. 4, 1388-1402, October 1999

[McIntyre and Hintz, 1998] G.A. McIntyre and K.J. Hintz, A Comparison of Several Maneuvering Target Tracking Models, SPIE Conference on Signal Processing, Sensor Fusion and Target Recognition, Vol. 3374, April 1998

[Ramachandra, 1993] K.V. Ramachandra, B.R. Mohan, B.R. Geetha, A Three State Kalman Tracker Using Position and Rate Measurements, IEEE Transactions on Aerospace and Electronic Systems, Vol. 29, No. 1, 215-222, January 1993

[Ramachandra, 2000] K.V. Ramachandra. Kalman Filtering Techniques for Radar Tracking, Marcel Dekker, New York, 2000

[Schutz, et al., 1997] Robert Schutz, Bruce Engleberg, Warren Soper, Richard McAllister, Combined Kalman Filter (CKF) and JVC Algorithms for AEW Target Tracking Applications, Proceeding of the SPIE Conference on Signal and Data Processing of Small Targets, Vol. 3163, 164-176, 1997

[Schutz, et al., 1999] Robert Schutz, Bruce Engleberg, Warren Soper, Richard McAllister, Maneuver Tracking Algorithms for AEW Target Tracking Applications, Proceeding of the SPIE Conference on Signal and Data Processing of Small Targets, Vol. 3809, 308-319, 1999 